\title{
Sports Injuries and Medicine
}

\section{Brain Motion, Deformation, and Potential Injury During Soccer Heading}

\author{
Charles F. Babbs*
}

Department of Biomedical Engineering Weldon School, Purdue University, West Lafayette, USA

"Corresponding author: Charles F. Babbs, Weldon School of Biomedical Engineering, Purdue University, West Lafayette, USA. Tel: +17654942995; Fax: +17654941193; Email: babbs@purdue.edu

Citation: Babbs CF (2018) Brain Motion, Deformation, and Potential Injury During Soccer Heading. Sports Injr Med: JSIMD-133. DOI: $10.29011 / 2576-9596.100033$

Received Date: 16 February, 2018; Accepted Date: 14 April, 2018; Published Date: 25 April, 2018

\begin{abstract}
This paper addresses the problem of what is happening physically inside the skull during head-ball contact. Mathematical models based upon Newton's laws of motion and numerical methods are used to create animations of brain motion and deformation inside the skull.

Initially a $1 \mathrm{~cm}$ gap filled with Cerebrospinal Fluid (CSF) separates the brain from the rigid skull in adults and older children. Whole head acceleration induces a pulse of artificial gravity within the skull. Because brain density differs slightly from that of CSF, the brain accelerates and strikes the inner aspect of the skull, undergoing viscoelastic deformation, ranging from 1 to 2 percent compression for normal heading with good technique, from 5 to 10 percent compression for normal heading with poor technique, and from 30 to 40 percent compression for accidental heading of rising balls at close range. The amount of local strain developed at the point of impact is magnified by force concentration caused by the mismatch between curvature of the brain and curvature of the overlying skull. Computed values are compared to a putative safe level of $1 \%$ maximum compressive strain permitted by the skull anatomy of woodpeckers.

This fresh biomechanical analysis allows one to visualize events within the skull during soccer heading. The results suggest heading safety is greatly improved when players head the ball with greater effective body mass, which is determined by a player's size, strength, and technique. Focus on teaching proper technique, re-design of age-appropriate balls with reduced weight, and strict avoidance of head contact with fast, rising balls kicked at close range can substantially reduce the risk of subtle brain injury.
\end{abstract}

Keywords: Acceleration; Biomechanics; Brain; Concussion; Football; Head; Heading; Injury; Player; Safety; Soccer; Strain; Trauma; Woodpecker

\section{Introduction}

Modern medicine abounds with examples of previously unappreciated harmful effects of repeated subtle injury to the body. Familiar examples include lung cancer caused by cigarette smoking, skin cancer caused by sun exposure, auditory nerve damage caused by loud noises, and brain damage caused by alcohol ingestion [1]. In the particular case of mechanical injury to the brain, shaken baby syndrome is now recognized as an important ill effect of head acceleration previously thought to be tolerable. Pugilistic dementia in prizefighters subjected to repeated blows to the head is a recognized medical condition [2]. Even mild concussions, when repeated, are now well documented to produce severe and lasting brain damage $[3,4]$.

The sport of soccer (known as football outside the United States) is unique in that the ball can be directed deliberately and purposefully with the head--a play that is termed "heading" the ball. A series of studies reported from Norway has suggested that brain atrophy upon CT scanning [5], electroencephalographic changes [6], and subtle deficits in memory, concentration, planning, and alertness $[7,8]$ occur in long-time soccer players that can be related to the number of balls headed. Soccer players may also suffer repeated mild concussions from collisions with other players, from high kicks, or from falling to the ground, which might possibly explain brain abnormalities in the absence of injurious effects of heading the ball $[9,10]$. Interestingly, Barnes [9] and Boden [11] studied the incidence of concussions in players from contact with 
objects other than the ball, such as the ground, a goal post, or another player. Both studies found the average incidence of such non-ball-related concussions to be 1 per 20 years of active playing. This incidence is much too low to explain repeated or lasting brain injury in typical soccer players [12], especially when two thirds of such concussions were mild, Grade I concussions, characterized by confusion without amnesia [9]. Hence one must seriously consider head-ball contact as a possible cause of injury, either in heading accidents, in which balls strike players unawares, or in normal heading that occurs in the routine course of a playing career.

Matser and coworkers $[7,13]$ found that Dutch amateur and professional soccer players performed significantly worse in tests of memory and planning than did control runners and swimmers. A follow up study [14] found that the number of headers in one season was related to poorer results on tests measuring focused attention and visual/verbal memory in a group of 84 active professional soccer players from several premier league soccer clubs. Lipton and coworkers [15] using diffusion-tensor magnetic resonance imaging in 37 soccer players who had headed the ball a median number of 432 times in the past year found significant statistical associations of heading with abnormal white matter microstructure and with poorer memory scores. In a similar study Koerte and coworkers [16] found scattered white matter abnormalities in the brains of young adult soccer players compared to the brains of swimmers. Another study of 19 players using transcranial magnetic stimulation [17] found that a single session of normal heading practice caused immediate, transient impairments in corticomotor function and memory. A study of high level youth players in the United States found 40 percent of those who frequently headed the ball had impaired IQ scores [18].

In contrast, a study of U.S. National team players with a long history of heading [19] found no evidence of brain injury compared with track athletes unexposed to heading. Also Kontos et al. [20]. studied verbal and visual memory, motor processing, and reaction times in youth soccer players and found no differences among low-, moderate-, and high-exposure header groups. The issue remains controversial and of substantial concern to parents of younger players who take up the sport early and may quickly advance to higher competitive levels, where heading is an encouraged and expected part of the game. These same players will be exposed to a lifetime of play and could be at risk of cumulative accelerationrelated brain injury. Considering the estimated 200 million active soccer players worldwide [13,21], even a small percentage risk of permanent brain injury would have serious public health implications.

Describing the biomechanics of closed head injury is a classic and still open problem that is difficult to study experimentally. Potentially harmful blunt impacts can last for as little as 2 to $200 \mathrm{msec}$ [22]. Subsequent motion of the brain inside the skull lasts just a few hundreds of milliseconds and is rarely seen. It is virtually impossible to study in real time under real field conditions. Only a few studies in animals, using high-speed photography through transparent plastic skull implants or using high speed fluoroscopy of implanted radiodense pellets [23-26] have examined the actual motion of the brain during closed head injury. Fortunately, mathematical analysis and modeling of the skull and brain in response to known pulses of head acceleration allow one to explore a wide variety of conditions that are difficult, impossible, or unethical to reproduce in animals or in humans [27]. This mathematical approach is ideal for visualizing what actually happens to the brain during and after heading a soccer ball. In this paper we use a validated finite element model to reveal the instantaneous patterns of deformation of the brain during the first 1.5 seconds after head-ball contact.

\section{Methods}

\section{Approach}

Unlike some prior biomechanical theories of closed head injury that do not explicitly include the subarachnoid space and Cerebrospinal Fluid (CSF) surrounding the brain [28-31], the present simulations are based on a validated computational model [27] describing the motion of the brain, suspended in cerebrospinal fluid, in response to "artificial gravity" caused by head acceleration. Here it is helpful to distinguish clearly two frames of reference for motion. The first is the internal frame of reference within the skull. This frame of reference is important because it is the relative motion of brain with respect to the rigid skull that is responsible for closed head injury. The second is the external frame of reference in which the head, neck, and body move. This frame of reference is important because an external blow or fall causes movement of the whole head in this frame. Acceleration of the head in the external frame produces acceleration of the brain in the internal frame in response to an apparent pulse of "artificial gravity" within the skull. The effect is like that felt by a man in a suddenly rising elevator. Within the internal frame of reference of the elevator, the passenger experiences added acceleration toward the floor of the elevator that is equal and opposite to the upward acceleration of the elevator in the shaft [32]. The effect of whole head acceleration on the brain is similar to the effect of acceleration of a rising elevator on its occupants. In this case the skull is analogous to the walls of the elevator, and the brain is analogous to a passenger in the elevator.

Linear acceleration of the rigid skull by an amount, $\mathrm{a}_{\text {head, }}$ produces a corresponding artificial gravity within the skull of $-\mathrm{a}_{\text {head. }}$ Unlike a passenger in an elevator, however, the brain is suspended in aqueous CSF. If $\rho_{\mathrm{CSF}}$ is CSF mass density, $\rho$ is brain mass density, and $\mathrm{V}$ is brain volume, then the force of artificial gravity on the brain is $\rho \mathrm{V}\left[-\mathrm{a}_{\text {head }}\right]$, and the buoyancy of the brain (from Archimedes' principle) is $-\rho_{\mathrm{CSF}} \mathrm{V}\left[-\mathrm{a}_{\text {head }}\right]$. Applying Newton's 
second law of motion (Force $=$ mass $\mathrm{x}$ acceleration) to the whole brain and ignoring drag",

$\left(\rho-\rho_{\mathrm{CSF}}\right) V\left[-a_{\text {head }}\right]=\rho V a_{\text {brain }}$,

where $\mathrm{a}_{\text {brain }}$ is the acceleration of the brain with respect to the skull in the direction parallel to whole head acceleration. In turn, the acceleration of the brain in the internal frame of reference of the skull,

$$
a_{\text {brain }}=-\frac{\left(\rho-\rho_{\mathrm{CSF}}\right)}{\rho} \mathrm{a}_{\text {head }} \text {. }
$$

This acceleration is substantially less than the oppositely directed acceleration, $a_{\text {head, }}$ of the head as a whole. If brain and CSF density were perfectly matched there would be zero acceleration of the brain with respect to the skull. In reality, CSF density is approximately 1.00 , and brain density is approximately 1.046 [33-35], so internal brain acceleration is about 5 percent of external head acceleration in magnitude.

\section{Brain Velocity Toward the Skull}

By Newton's third law of motion, the reactive force on the head is equal in magnitude and opposite in direction from the force that the head exerts on the ball to do work: $\overrightarrow{\mathrm{F}}_{\text {head }}=-\overrightarrow{\mathrm{F}}_{\text {ball }}$. By Newton's second law of motion, the product of the average reactive force and the brief time interval, $\Delta \mathrm{t}$, of the collision equals the mass of the ball multiplied by the change in velocity of the ball:

$\overrightarrow{\mathrm{F}} \Delta \mathrm{t}=\mathrm{m}_{\text {ball }} \Delta \overrightarrow{\mathrm{v}}_{\text {ball }}=-\hat{\mathrm{m}}_{\mathrm{p}} \Delta \overrightarrow{\mathrm{v}}_{\text {head }}$, where the player's effective mass, $\hat{\mathrm{m}}_{\mathrm{p}}$, is that of the head anchored by the neck to the torso. Hence

$\mathrm{m}_{\text {ball }} \Delta \overrightarrow{\mathrm{v}}_{\text {ball }}=-\hat{\mathrm{m}}_{\mathrm{p}} \Delta \overrightarrow{\mathrm{v}}_{\text {head }}$,

Note: ${ }^{\#}$ It is reasonable to ignore the effects of viscous drag on the brain from CSF water, because the distance traveled by the brain through CSF is short and brain speeds relative to the skull are limited over this distance, and in turn

$\mathrm{a}_{\text {head }}=\frac{\Delta \overrightarrow{\mathrm{v}}_{\text {head }}}{\text { At }}=-\frac{\mathrm{m}_{\text {ball }}}{\hat{\mathrm{m}}_{\mathrm{p}} \Delta \mathrm{t}} \Delta \overrightarrow{\mathrm{v}}_{\text {ball }}$.
For a ball that is headed back in the direction from which it came, we can estimate the change in velocity of the head and supporting torso from the change in velocity of the ball, the mass of the ball, and the "effective mass" of the player.

To compute the change in velocity of the brain toward the skull within the internal frame of reference of the skull, we have, using Equation (2)

$\mathrm{v}_{0}=\mathrm{a}_{\text {brain }} \Delta \mathrm{t}=-\mathrm{a}_{\text {head }} \frac{\left(\rho-\rho_{\mathrm{CSF}}\right)}{\rho} \Delta \mathrm{t}$.

Substituting for head acceleration from Equation (4),

$\mathrm{v}_{0}=-\frac{\mathrm{m}_{\text {ball }}}{\hat{\mathrm{m}}_{\mathrm{p}}} \cdot \frac{\left(\rho-\rho_{\mathrm{CSF}}\right)}{\rho}\left|\Delta \overrightarrow{\mathrm{v}}_{\mathrm{b}}\right|$

which is the initial velocity of the brain toward the skull in terms of the change in velocity of the ball, the ball/player mass ratio, and the brain minus CSF density ratio.

\section{Ball Sizes and Weights}

Ranges for the masses, diameters, and recommended inflation pressures for youth and adult size soccer balls are available from the manufacturers and from the Laws of the Game [36,37]. Masses of soccer balls of various sizes are given in Table 1,

\begin{tabular}{|c|c|c|}
\hline Ball size & Age (years) & Mass (grams) \\
\hline 3 & $6-8$ & $310-340$ (midrange 325) \\
\hline 4 & $9-11$ & $310-370$ (midrange 340) \\
\hline 5 & Adult & $400-450$ (midrange 425) \\
\hline
\end{tabular}

Table 1: Masses of soccer balls of various sizes.

\section{Horizontal Ball Velocities}

Horizontal ball speeds, including goal kicks, corner kicks, crosses, clearances, and shots, in youth, teen, and adult soccer games [38] have been measured by the author with suitable accounting for the effects of air resistance on the motion of the ball. Figure 1 illustrates average trajectories calculated for lofted balls. Data points indicate equal time points, separated by $0.1 \mathrm{sec}$; hence the distance between data points on the trajectory curves is related to a ball's speed. 


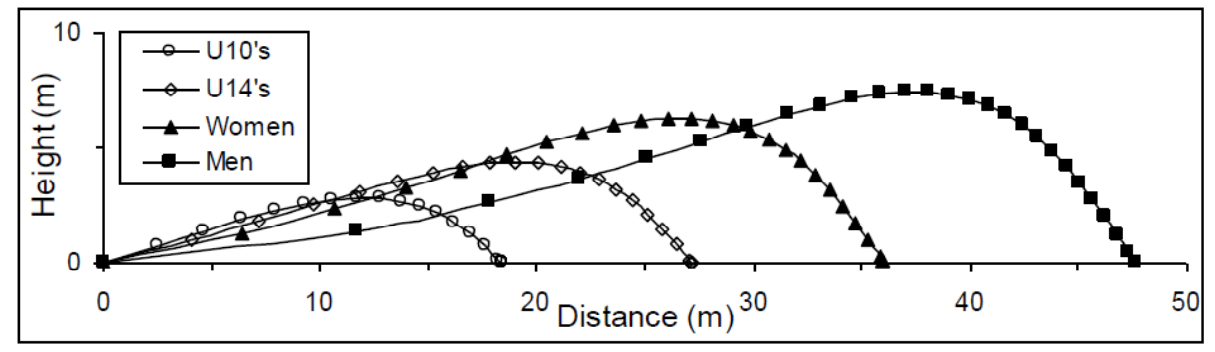

Figure 1: Calculated average trajectories of lofted balls observed in soccer games. Curves show non-parabolic shapes because of air resistance. Data points are equally spaced in time, $0.1 \mathrm{sec}$ apart. U10's indicates under 10 1/2 year-old players. U14's indicates under 14 1/2 year-old players [38].

As the size and strength of the player's increases, the distances covered and initial velocities of balls increase, as expected. However, air resistance, which is related the square of velocity, also increases and has a longer time to act as balls travel farther. As a result, the horizontal component of velocity, which is most relevant to heading safety, becomes similar for all types of lofted balls on the descending limb of flight, regardless of the age and strength of the players. However, rising balls kicked by adult players at close range are exceedingly fast, with initial horizontal velocities of 25 to $75 \mathrm{~m} / \mathrm{sec}$ for adult players, in keeping with previous reports $[15,17]$. Figure 2 illustrates the statistical distributions of descending horizontal ball velocities at head height for both male and female players ages 10 through adult in 16 competitive games. The values range between 1 and $14 \mathrm{~m} / \mathrm{sec}$.

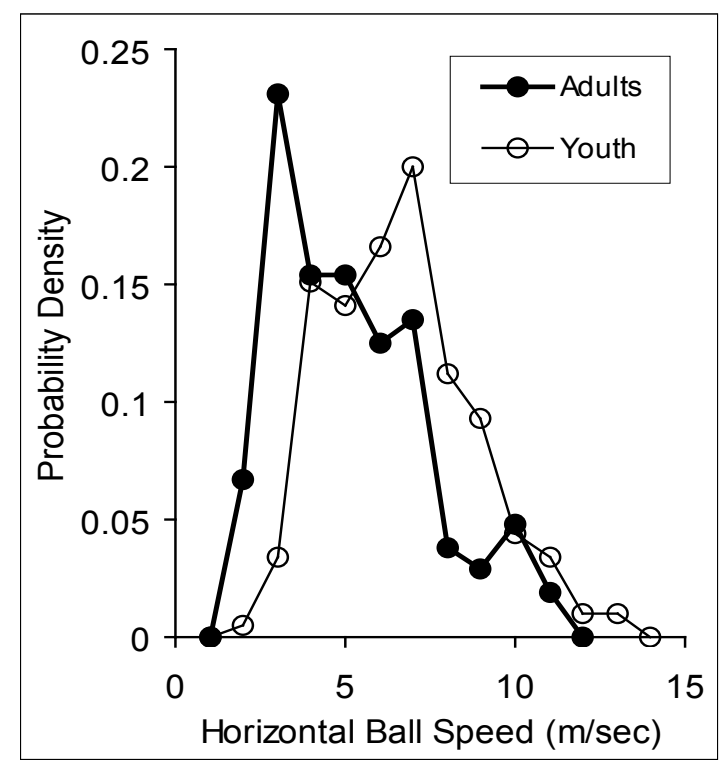

Figure 2: Distributions of calculated horizontal ball speeds for falling balls at head height obtained from spherical projectile formulas using time and distance data recorded during 16 competitive games [38]. The adult distribution represents 104 balls that could have been headed. The youth distribution represents 205 balls that could have been headed.

\section{Effective Mass of the Player}

It is wrong to consider the head as a mass unconnected to the body, since players with even moderately effective training learn to tense the neck muscles to put more effective mass behind the ball. However, it is also wrong to assume the player's entire body mass is lumped behind the ball during heading. The ball is struck by the standing or jumping player at one end of a long lever arm, which is free to rotate backwards either at the point of contact with the ground or in free space (Figure 3). In the case of grounded players it is useful to the regard the player as a slab-like mass that is free to rotate at the point of foot contact with the turf. In the case of jumping players, it is useful to regard the nlaver as a slab-like mass that is free to rotate about its center of $\mathrm{Fx}_{\mathrm{x}} \sin \theta$

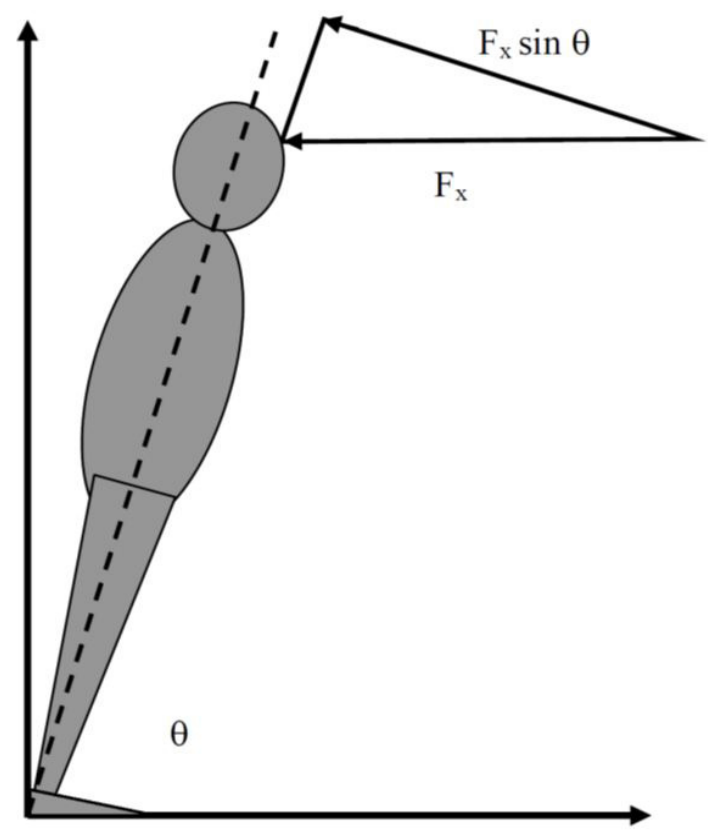

Figure 3: Model of a grounded player. $F_{x}$ indicates the horizontal component of reactive force acting on the head during head-ball impact. $\mathrm{F}_{\mathrm{x}} \sin \theta$ is the force component perpendicular to the long axis of the body. 
The concept of the effective mass of an object with a linearly distributed mass density is well precedented in physics [25,26]. Consider a scenario in which a player running due east strikes a ball that is moving due west with the forehead, and consider the horizontal components of head and ball motion. The player can be regarded as a single east moving "effective mass" that collides with the west moving ball to change its motion. As shown in Appendix 1 , the effective mass depends upon the angle, $\theta$, of inclination of the player (Figure 3). Suppose that the player uses excellent technique, with the neck strong and stiff, so that the head does not wobble backwards significantly. In this case the effective mass of a player at attack angle, $\theta$, is

$\hat{\mathrm{m}}_{\mathrm{p}}=\frac{\mathrm{m}_{\mathrm{p}}}{2 \sin ^{2} \theta}$

for a grounded player and

$\hat{\mathrm{m}}_{\mathrm{p}}=\frac{\mathrm{m}_{\mathrm{p}}}{2 \sin ^{2} \theta+\cos ^{2} \theta}$

for a jumping player. For example, the effective mass for player standing vertically $(\theta \approx 90$ degrees, $\sin (\theta) \approx 1)$ is roughly one half the body mass for either a grounded or jumping player model. The effective mass for a diving header $(\theta \approx 0$ degrees, feet off ground, $\sin (\theta) \approx 0, \cos (\theta) \approx 1$ ) is roughly 100 percent of the body mass, as expected. Here, for simplicity, an effective mass of one half total body mass is used to model a player using ideal heading technique, in which the player attacks the ball with neck muscles tensed so that entire body mass is connected to the ball during impact. To model the worst possible heading technique the mass of the head only is used. The mass of the head alone is in the range of 2 to $5 \mathrm{~kg}$, depending on age (see Table 3) [27]. For any particular trajectory of an incoming ball, the effective mass of the player is a major determinate of head acceleration at impact. The greater the effective mass of the player, the smaller is the acceleration of the head.

\section{Analytical Model of the Brain and Skull}

In their 1994 textbook, The Mechanisms of Continua and Wave Dynamics, Brekhovskikh and Gancharov [39] describe collision of a column of elastic material impacting a rigid wall at constant velocity, $\mathrm{v}_{0}$, in one dimension. For a column comprised of a material of density, $\rho$, having uniform stiffness (Young's modulus of elasticity), E , and hitting a solid wall with initial velocity, $\mathrm{v}_{0}$, behaves as shown in Figure 4. After initial contact with the wall a wave of compressive strain is propagated through the column, until it comes to a complete stop. At this point there is maximal compression, which is then followed by rebound of the material in a last-in/first-out pattern. The maximum compressive strain, just prior to rebound, is a good index of overall brain deformation following sudden acceleration of the whole head [27,39]. Without elaborating details of strain wave propagation [39], one may easily compute maximal compression from considerations of conservation of energy, as follows.

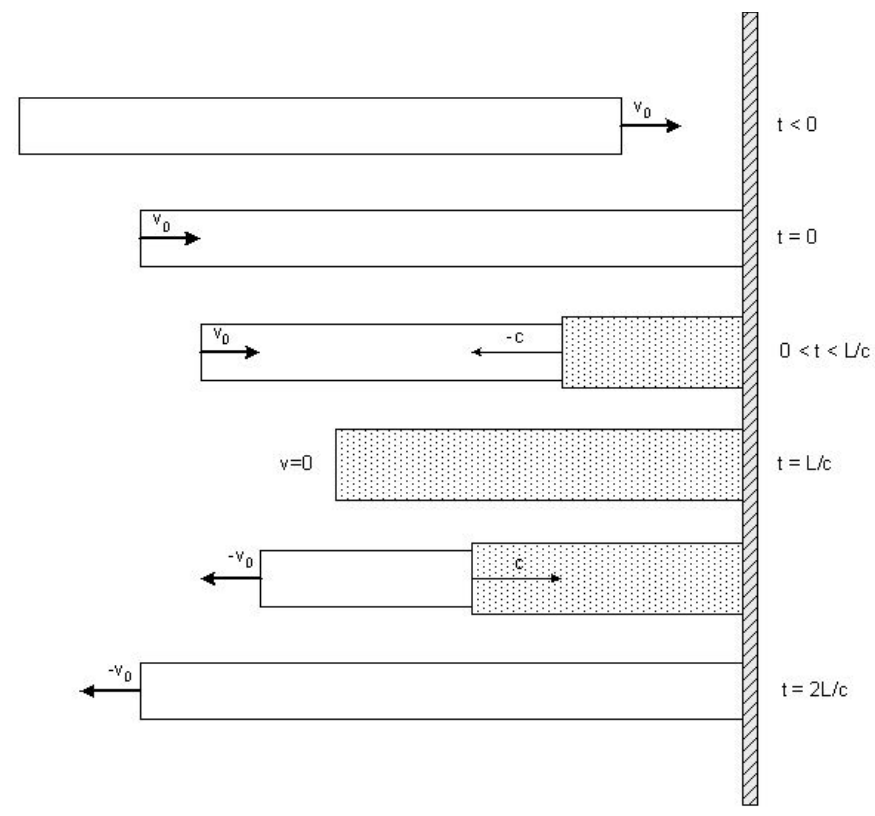

Figure 4: Phases of motion of an elastic column hitting a solid wall with initial velocity, $\mathrm{v}_{0}$. Propagation of a compressive strain wave is shown at successive times after impact. The initial length of the bar is $\mathrm{L}$. The strain wave velocity is $c=\sqrt{E / \rho}$. Typically, $c>>v_{0}$.

In cases of minimal damping the incoming kinetic energy of the brain is equal to the potential energy of the compressed column at the instant just before rebound (no motion), so that

$\frac{1}{2} \mathrm{mv}_{0}^{2}=\frac{1}{2} \mathrm{k}(\Delta \mathrm{L})^{2}$

where $\mathrm{k}$ is the spring constant of the elastic column, and $\Delta \mathrm{L}$ is the change in length at maximum compression. Now, substituting for the spring constant, $\mathrm{k}$, as classically described [40], and for the mass, $\mathrm{m}$, we have

$$
\mathrm{k}=\frac{\mathrm{EA}}{\mathrm{L}_{0}}
$$

and 
$\mathrm{m}=\rho \mathrm{AL}_{0}$,

where E is Young's modulus of elasticity, A is the cross sectional area, $L_{0}$ is the original unstressed length of the elastic body, and $\rho$ is the mass density of the body.

Then by conservation of energy

$\frac{1}{2} \rho \mathrm{L}_{0} \mathrm{Av}_{0}^{2}=\frac{1}{2} \frac{\mathrm{EA}}{\mathrm{L}_{0}}(\Delta \mathrm{L})^{2}$

so that squared maximal strain is

$\varepsilon_{\max }^{2}=\frac{(\Delta \mathrm{L})^{2}}{\mathrm{~L}_{0}^{2}}=\frac{\rho}{\mathrm{E}} \mathrm{v}_{0}^{2}$,

and the maximal compressive strain, $\left(\mathrm{L}_{0}-\mathrm{L}\right) / \mathrm{L}_{0}$, is

$\varepsilon_{\max }=v_{0} \sqrt{\frac{\rho}{E}}$.

Here $\mathrm{v}_{0}$ represents the initial velocity of the brain approaching the skull within the internal frame of reference of the brain, accounting for the buoyancy of the brain in CSF. During isovolumic deformation $($ Poisson's ratio $=0.5)$ the expansive strain, $\left(\mathrm{w}-\mathrm{w}_{0}\right) / \mathrm{w}_{0}$, for width,

$\mathrm{W}$, perpendicular to $\mathrm{v}_{0}$, is equal to $\varepsilon_{\mathrm{z}}=0.5 \varepsilon_{\max }=0.5 \mathrm{v}_{0} \sqrt{\frac{\rho}{\mathrm{E}}}$. These values of $\varepsilon_{\max }$ and $\varepsilon_{\mathrm{z}}$ can be used to obtain an initial estimate of the maximal brain deformation as well as for validation of computer code representing more complex situations.

(a)

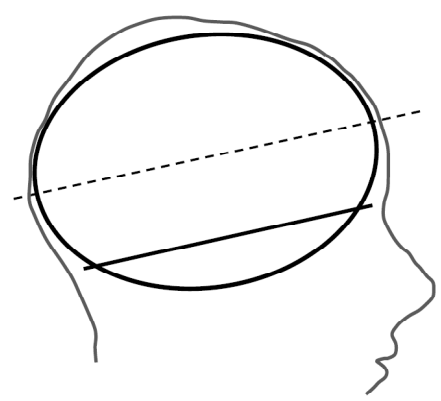

(b)

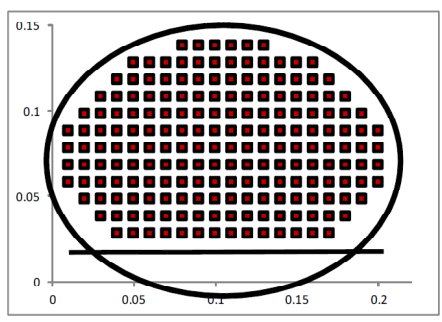

Figure 5: (a) Outline of skull, nose, face, and neck from a normal paramedian sagittal computed tomographic scan (gray) and superimposed elliptical model of skull (black). Dashed line represents the long, $\mathrm{x}$-axis of the model. If the player is leaning forward slightly, the long axis of the ellipse is approximately horizontal. (b) Sketch of finite element model of skull, brain, and surrounding CSF.

\section{Finite Element Model of the Brain and Skull}

To explore brain motion and deformation in more detail, a two-dimensional model of the rigid skull is defined in the shape of a truncated ellipse, as shown in Figure 5. If the player is leaning forward slightly when heading, the long axis of the ellipse is approximately horizontal. Within this rigid container a twodimensional model of a viscoelastic brain is defined by rows and columns of finite elements in the shape of a similarly truncated ellipse with major and minor axes $0.9 \mathrm{~cm}$ smaller than those of the model skull, the $0.9 \mathrm{~cm}$ gap representing the CSF-filled subarachnoid space. This shape represents a paramedian section on either side through the frontal and occipital lobes of the cerebral cortex (Figure 5), surrounded by a $0.9 \mathrm{~cm}$ gap filled with CSF.

The viscoelastic brain model can be accelerated parallel to its long axis and also rotated in the sagittal plane within the CSF containing subarachnoid space. Specific dimensions and tissue parameters for the various geometric models of the brain are indicated in Table 2. The model brain is treated as a uniform viscoelastic mass of homogenous density and elasticity, in keeping with experimental observations [33,41]. The model brain is isovolumic (i.e. Poisson's ratio $=0.5$ ), so that neither brain volume nor CSF volume changes during or immediately after impact. Viscoelastic properties of brain are those previously described [27]. The maximal internal fronto-occipital diameter of the human skull is taken as $20.8 \mathrm{~cm}$ for an adult and also for children over 7 years of age [42].

\begin{tabular}{|c|c|}
\hline Property (units) & $\begin{array}{c}\text { Numerical } \\
\text { values }\end{array}$ \\
\hline Front to back brain diameter $(\mathrm{cm})$ & 19 \\
\hline Top to bottom brain diameter $(\mathrm{cm})$ & 13 \\
\hline $\begin{array}{c}\text { Unstressed horizontal and vertical spacing } \\
\text { between adjacent nodes }(\mathrm{cm})\end{array}$ & 10000 \\
\hline Young's modulus of brain tissue (Pascals) & 100 \\
\hline Damping modulus of brain tissue (Pascal-sec) & 1046 \\
\hline Brain density $\left(\mathrm{kg} / \mathrm{m}^{3}\right)$ & 1000 \\
\hline CSF density $\left(\mathrm{kg} / \mathrm{m}^{3}\right)$ & \\
\hline
\end{tabular}

Table 2: Parameters of the standard finite element model [27].

The model viscoelastic brain is regarded as a modified Voigt/ Maxwell body [43] including discrete mass elements connected by both springs and dampers in a rectangular 8-way mesh. The 
mathematics are specifically designed for strain analysis with significant relative motion of the elements. An initial rectangular matrix of masses is trimmed or sculpted to represent a truncated ellipse in two dimensions. The 8 neighbors of an indexed mass $\mathrm{m}_{\mathrm{i}, \mathrm{j}}$ are denoted N, E, S, W, for north, east, south, and west, and the diagonal neighbors are denoted NE, NW, SE, and SW. Each mass is connected to neighbors $\mathrm{N}, \mathrm{E}, \mathrm{S}, \mathrm{W}$ by a massless springs having spring constant, $\mathrm{k}$, and parallel dampers having damping constant, $\mu$. On the diagonals each mass is connected to neighbors NE, NW, SE, and SW by massless springs having spring constant, $\mathrm{k}_{\mathrm{d}}$, and parallel dampers of damping constant, $\mu_{\mathrm{d}}$. At rest the component masses are spaced distance, $\mathrm{d}_{0}=1.0 \mathrm{~cm}$, apart in the N-S and E-W

dimensions and by distance $\sqrt{2} \mathrm{~d}_{0}=1.41 \mathrm{~cm}$ on the diagonals.

The value of each interior mass is $\mathrm{m}=\rho \mathrm{d}_{0}^{3}$. Edge masses have value $\mathrm{m} / 2$ and corner masses have value $\mathrm{m} / 4$. Further details are provided in reference [27].

\section{Numerical Methods}

In the present research we wish to track the motion of each node in time during and after a brief forceful acceleration of the head. For an interior mass $\mathrm{i}, \mathrm{j}$ the $\mathrm{x}$-component of acceleration depends upon the sum of applied forces due to local artificial gravity from whole head acceleration, from neighboring springs, and from neighboring dampers, which, using "double dot" notation for the acceleration, is given by

$\mathrm{m}_{\mathrm{i}, \mathrm{j}} \ddot{\mathrm{x}}_{\mathrm{i}, \mathrm{j}}=\mathrm{F}_{\mathrm{xg}}+\mathrm{F}_{\mathrm{xk}}+\mathrm{F}_{\mathrm{x} \mu}$.

The orthogonal (y) component of acceleration is given by

$m \ddot{y}_{i, j}=F_{y g}+F_{y k}+F_{y \mu}$.

The small effect of ordinary gravity from the Earth's mass is ignored, being much smaller in magnitude than head acceleration during impact. These expressions and the similar ones for edge nodes of mass $\mathrm{m} / 2$ and for corner nodes of mass $\mathrm{m} / 4$ can be solved for the acceleration of each node in the array as described in detail in reference[27]. Once these complexities of the force components are dealt with and the $\mathrm{x}$ and $\mathrm{y}$ components of acceleration for each node are known, the net accelerations are doubly integrated using a simple Euler method to extrapolate from time $t$ to time $t+\Delta t$, namely

$\dot{\mathrm{x}}(\mathrm{t}+\Delta \mathrm{t})=\ddot{\mathrm{x}}(\mathrm{t}) \Delta \mathrm{t}$,

$\mathrm{x}(\mathrm{t}+\Delta \mathrm{t})=\mathrm{x}(\mathrm{t})+\dot{\mathrm{x}}(\mathrm{t}) \Delta \mathrm{t}$,

and similarly for the time derivative, $\dot{\mathrm{y}}(\mathrm{t}+\Delta \mathrm{t})$, and $\mathrm{y}(\mathrm{t}+\Delta \mathrm{t})$, beginning with suitable initial conditions.

Results are displayed in computer animations showing motion of all nodes in the model brain as a function of time. The time step of integration, $\Delta t$, is selected such that doubling or halving the time step does not change the results. Motion of the nodes is constrained when they hit the wall of the surrounding skull. To deal with sharp points on outside corners of the rectangular grid of the finite element model during brain-skull collisions, interpenetration of the outside corners of the brain past the skull boundary for a distance of $\mathrm{d}_{0} / 2$ is allowed before motion is stopped. This correction eliminates high strain artifacts caused by spatial discretization of the edges in of the model in the $\mathrm{x}$ - and $\mathrm{y}$-dimensions.

\section{Making Animations}

The forgoing numerical model was implemented in Visual Basic code within an Excel spreadsheet on ordinary personal computer. To visualize motion of a generalized $2 \mathrm{D}$ model of the brain at times $t>0$ the $x-y$ positions of all nodes are plotted at equally spaced sample times to create a series of "movie frames", creating an animation.

\section{Indices of Brain Deformation}

For simplicity a reasonable summary metric for local compressive deformation during brain-skull collisions in the setting of soccer heading is computed as the $\mathrm{x}$-axis compressive strain between nodes of the finite element model, namely

$\varepsilon_{\mathrm{x}}=1-\frac{|\mathrm{x}(\mathrm{n}+1, \mathrm{~m})-\mathrm{x}(\mathrm{n}, \mathrm{m})|}{\mathrm{d}_{0}}$.

If the distance between nodes equals the unstressed, resting distance, $\mathrm{d}_{0}$, then $\varepsilon_{\mathrm{x}}=0$. If the distance between nodes is less than $\mathrm{d}_{0}$, then $\varepsilon_{\mathrm{x}}$ increases, indicating compressive strain. In this particular problem the simple major axis compressive strain mirrors the more complex Von Mises strain [44] as a scalar metric of local brain deformation. As an index of potential injury, the maximal value of $\varepsilon_{\mathrm{x}}$ across all nodes in the model, $\varepsilon_{\mathrm{xmax}}$, is plotted as a function of time after impact.

\section{Results}

\section{Range of Models Tested}

Table 3 shows standard models of adult players, Under 14year old age group players (U14) and Under10-year old age group players (U10). Soccer ball masses for the three groups are based on median values of Size 5 balls for adults, Size 4 balls for U14 players, and size 3 balls for U10 players, as shown in Table 1. For each age group, good heading technique and poor heading technique models are created using the effective mass of the head and torso. The effective mass representing good technique is chosen as one half 
the average total body mass. In this case the player tenses the neck and upper torso and actively bends the torso toward the ball at the time of contact, increasing the effective mass behind the ball. The effective mass representing poor technique is chosen to represent the approximate mass of the whole head and neck, when minimal muscle tone acts to semi-rigidly connect the head and neck with the torso of the player. Three categories of ball velocities represent median speed lofted balls and the fastest lofted balls recorded from actual games including players of different ages (Figure 2), as well as estimates of rising ball velocities off the foot at close range.

\section{Maximum Compressive Strains in Analytical Models}

Table 3 also shows in the rightmost column the maximal compressive strains calculated for the simple analytical model of Figure 4 using the conservation of energy approach as derived in Equations (7) through (11). For all age groups using good technique to head lofted passes with either average or maximal speed, no more than 1 percent overall compression of the brain occurs during heading. In the analytical models of poor technique for all age groups, using age-appropriate balls, 3 to 6 percent compression of the brain occurs during heading. A similar range of compressive strain occurs in models of good technique applied to close range rising balls.

More alarming compressions occur in models of unprepared or accidental heading of close range rising balls, as would occur in blocking a shot on goal with the head unprepared, or occasionally in practice sessions or games when a player is struck accidentally in the head by ball kicked by another player (Models 6, 12, and 18 in Table 3). This situation can occur in training when multiple players are shooting at goal with multiple balls, or in games when a defender quickly clears a ball from in front of goal, striking another player accidentally in the head. For adults, teens, and youth players the average, whole-brain compressive strains are over $30 \%, 20 \%$, and $13 \%$, respectively. Such impacts are not common but can occur several times in a playing career (including that of the author).

\begin{tabular}{|c|c|c|c|c|c|c|c|}
\hline Model \# & & $\begin{array}{r}\text { Ball } \\
\text { mass }(\mathrm{kg})\end{array}$ & ique & \multicolumn{2}{|c|}{$\begin{array}{l}\text { Effective Ball + player } \\
\text { mass }(\mathrm{kg}) \text { speed }(\mathrm{m} / \mathrm{sec})\end{array}$} & \multicolumn{2}{|c|}{$\begin{array}{l}\text { Internal v0 } \\
\quad(\mathrm{m} / \mathrm{sec}) \text { Max strain }\end{array}$} \\
\hline 1 & Adult & 0.425 & good & 35 & 8 & 0.004 & 0.005 \\
\hline 2 & Adult & 0.425 & poor & 5 & 8 & 0.031 & 0.032 \\
\hline 3 & Adult & 0.425 & good & 35 & 13 & 0.007 & 0.007 \\
\hline 4 & Adult & 0.425 & poor & 5 & 13 & 0.051 & 0.052 \\
\hline 5 & Adult & 0.425 & good & 35 & 76 & 0.042 & 0.043 \\
\hline 6 & Adult & 0.425 & poor & 5 & 76 & 0.297 & 0.304 \\
\hline 7 & U14 & 0.34 & good & 30 & 8 & 0.004 & 0.004 \\
\hline 8 & U14 & 0.34 & poor & 4 & 8 & 0.031 & 0.032 \\
\hline 9 & U14 & 0.34 & good & 30 & 13 & 0.007 & 0.007 \\
\hline 10 & U14 & 0.34 & poor & 4 & 13 & 0.051 & 0.052 \\
\hline 11 & U14 & 0.34 & good & 30 & 51 & 0.027 & 0.027 \\
\hline 12 & U14 & 0.34 & poor & 4 & 51 & 0.199 & 0.204 \\
\hline 13 & U10 & 0.325 & good & 20 & 8 & 0.006 & 0.006 \\
\hline 14 & U10 & 0.325 & poor & 3 & 8 & 0.040 & 0.041 \\
\hline 15 & U10 & 0.325 & good & 20 & 13 & 0.010 & 0.010 \\
\hline 16 & U10 & 0.325 & poor & 3 & 13 & 0.065 & 0.066 \\
\hline 17 & U10 & 0.325 & good & 20 & 26 & 0.019 & 0.020 \\
\hline 18 & U10 & 0.325 & poor & 3 & 26 & 0.130 & 0.133 \\
\hline
\end{tabular}

Table 3: Model parameters and maximal compressive strains.

\section{Brain Motion and Deformation in Finite Element Models}
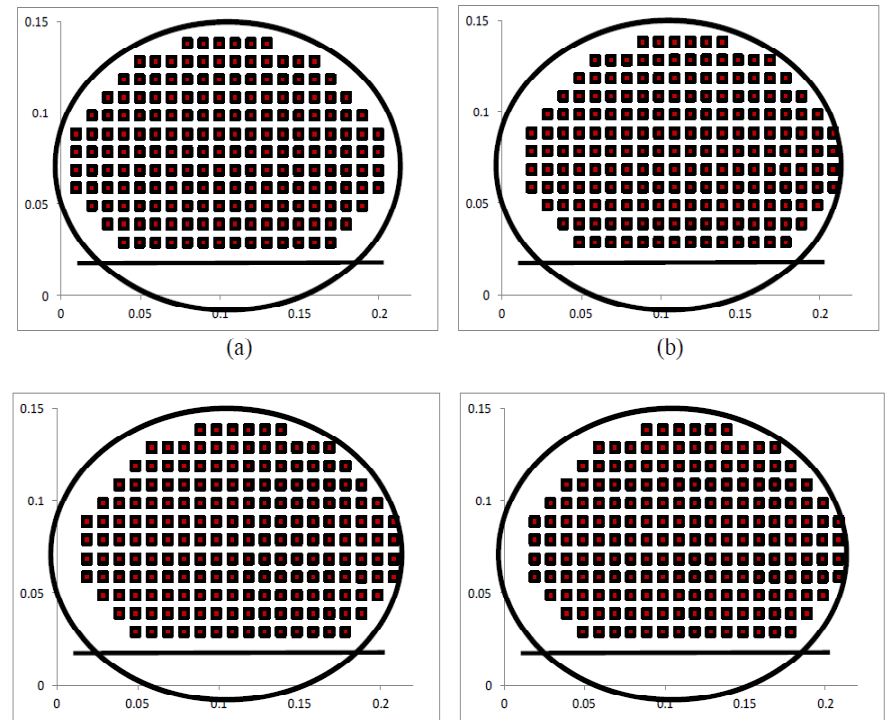

(c)

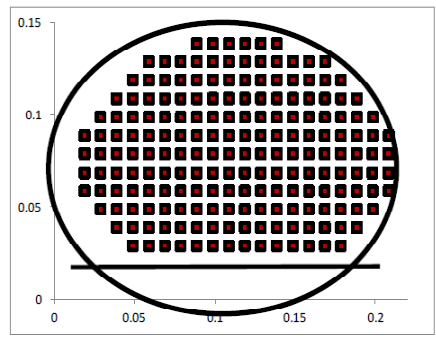

(d)

Figure 6: Snapshots of animations for normal heading of median speed balls. Axis scales are in meters. (a) $t=0$, initial state; (b) Adult Model 1, $t$ $=1.11 \mathrm{sec}$, near time of maximal compression after collision of the brain with the inner surface of the skull; (c) U14 Model 7 at $\mathrm{t}=1.2 \mathrm{sec}$ near time of maximal compression; (d) U10 Model 13 at $\mathrm{t}=0.84 \mathrm{sec}$ near time of maximal compression.

Figure 6(a) shows the numerical brain and skull model in its unstressed resting state. This model represents a front-to-back, paramedian slice through either hemisphere. The filled rectangles represent masses or nodes of a Voigt model, connected to each other by parallel springs and dampers (not shown) in an 8-way mesh, as described in detail previously [27]. In the unstressed state the nodes are $1 \mathrm{~cm}$ apart horizontally and vertically. The coarseness of the mesh represents the organization of the cerebral cortex into gyri and sulci, with a somewhat lumpy gross surface texture.

Figures 6(b) through 6(d) represent results in models of normal heading of medium speed lofted balls. Figure 6(b) shows one instantaneous state of the Model 1, representing an adult player heading an average speed descending Size 5 lofted ball with good technique. Ball contact has occurred at the right side of the skull. The brain has moved forward, toward the point of impact. The brain has collided with the skull at $4 \mathrm{~mm} / \mathrm{sec}$ (Table 3 ). The snapshot illustrates a moment in time (1.11 sec after impact) at maximal compression. There is little visible distortion. The maximal local compressive strain in the x-dimension is 1.29 percent. Figure 6(c) shows the maximal compression state of Model 7, representing a U14 player heading an average speed Size 4 ball. The maximal compressive strain is 1.21 percent. Figure 6(d) shows the state of Model 13, representing a U10 player heading a typical Size 3 ball. The maximal compressive strain is 1.37 percent. 
If the same adult, U14, and U10 players head the same median velocity balls with poor technique, allowing the head to wobble at impact, the sequences shown in Figures 7, 8, and 9 occur. Within a fraction of a second after impact brain skull contact occurs, producing modest visible compression and potential "coup" injury (b). The viscoelastic brain then rebounds and moves in the opposite direction, subsequently impacting the opposite pole of the skull, producing a second region of modest compression at the opposite pole and potential "contrecoup" injury (d). Maximum strains for adult, U14, and U10 players, respectively are 5.37, 5.37, and 6.32 percent

Maximal compressive strains for the first collisions in these finite element models are roughly twice the analytical values in Table 3. An explanation for this discrepancy is that only part of the brain is making contact with the skull, namely the mid-latitude regions in the models of Figures 7 through 9. A partial strip of tissue is absorbing the momentum of the entire brain, as the full brain comes to a complete stop and reverses direction, with only part of the frontal pole (or occipital pole in contrecoup) making contact with the skull. The amount of local strain developed near the point impact is increased by force concentration that is caused by the mismatch between the curvature of the brain and the curvature of the overlying skull. In contrast, for the analytical calculations in Table 3 using the simple one-dimensional model, the entire leading surface makes contact simultaneously and participates in deceleration of the whole brain.

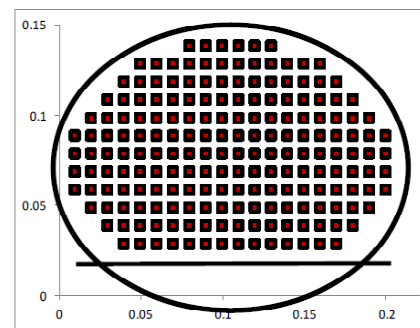

(a)

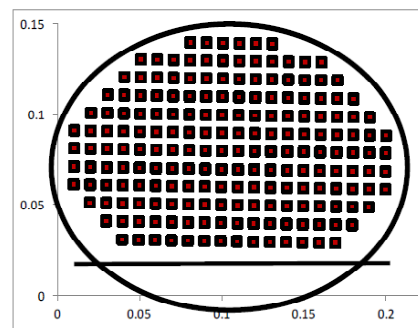

(c)

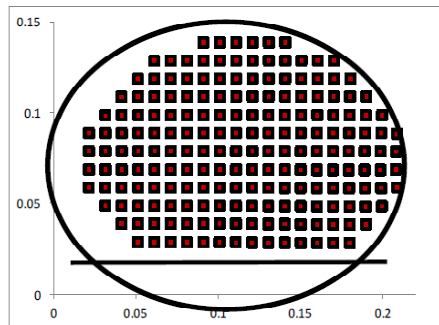

(b)

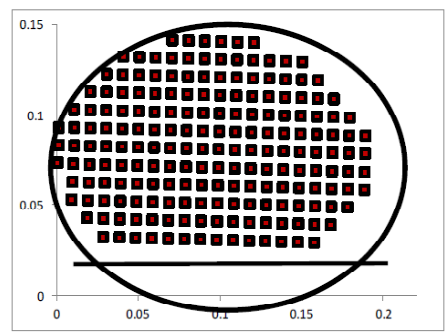

(d)
Figure 7: Snapshots of animations for adult Model 2. (a) $t=0$, initial state; (b) $\mathrm{t}=0.22 \mathrm{sec}$, coup; (c) $\mathrm{t}=0.5 \mathrm{sec}$, rebound;(d) $\mathrm{t}=0.8 \mathrm{sec}$, contrecoup.

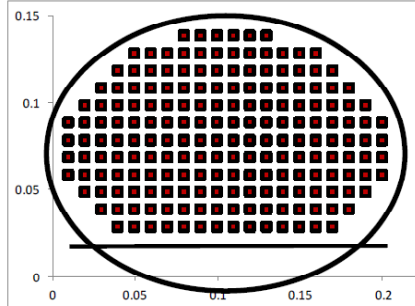

(a)

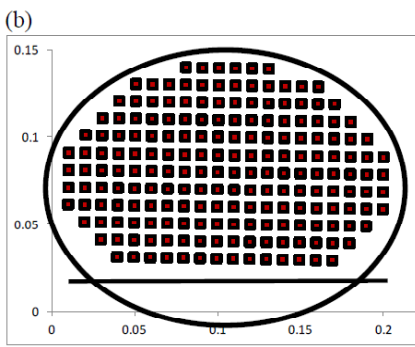

(c) (b)

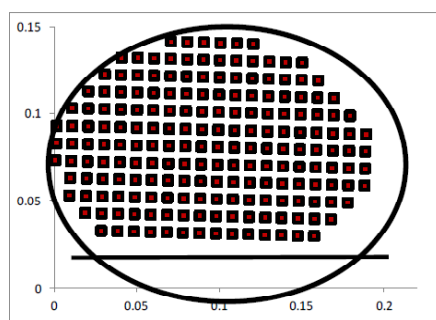

(d)

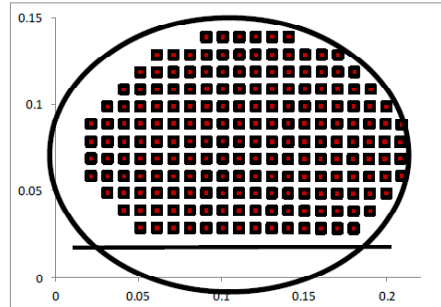

Figure 8: Snapshots of animations for U14 Model 8. (a) $t=0$, initial state; (b) $\mathrm{t}=0.23 \mathrm{sec}$, coup; (c) $\mathrm{t}=0.5 \mathrm{sec}$, rebound; (d) $\mathrm{t}=0.8 \mathrm{sec}$, contrecoup.

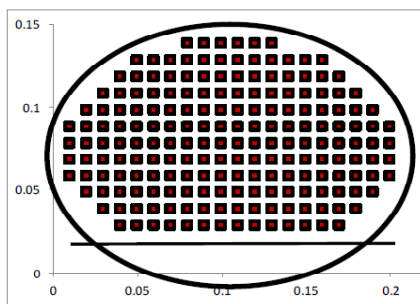

(a)

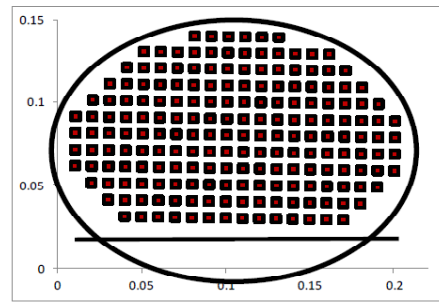

(c)

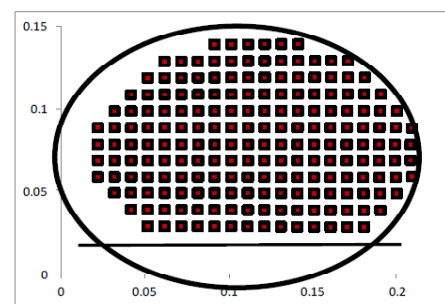

(b)

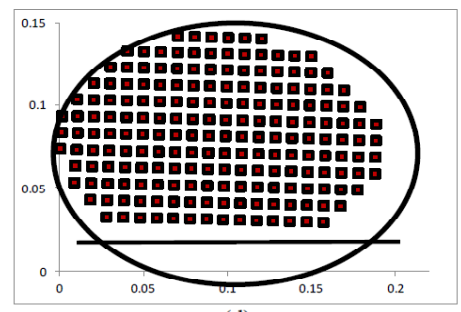

(d)
Figure 9: Snapshots of animation for U10 Model 14. (a) $t=0$, initial state; (b) $\mathrm{t}=0.17 \mathrm{sec}$, coup; (c) $\mathrm{t}=0.42 \mathrm{sec}$, rebound; (d) $\mathrm{t}=0.68 \mathrm{sec}$, contrecoup. Axis scales in meters.

Figure 10 (solid curves) illustrates the patterns of maximal compressive strain as a function of time for Models 2, 8, and 14 in Table 3. Alternate peaks represent coup and contrecoup impacts. The very small fluctuations between peaks represent internal jiggle. Reduced amplitude and speed with successive peaks represents viscous energy absorption by dampers. 

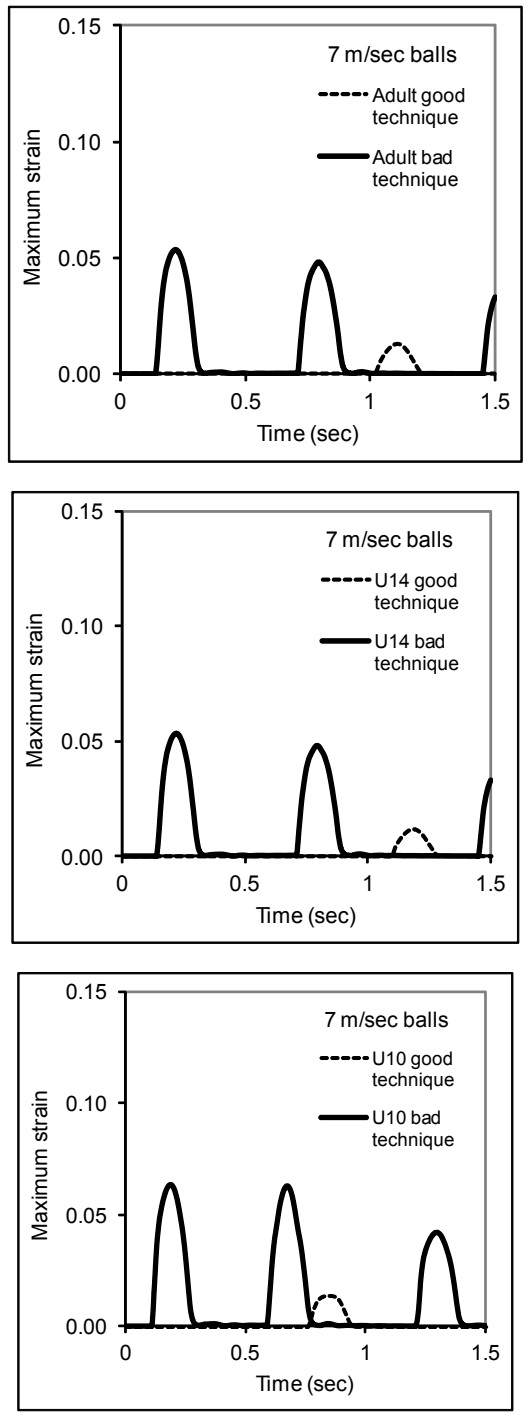

Figure 10: Time domain records of maximal compression strain in brains of players of various ages heading typical lofted balls; (top) Models 1 and 2; (middle) Models 7 and 8; (bottom) Models 13 and 14.

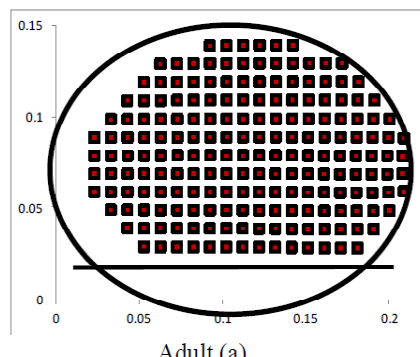

Adult (a)
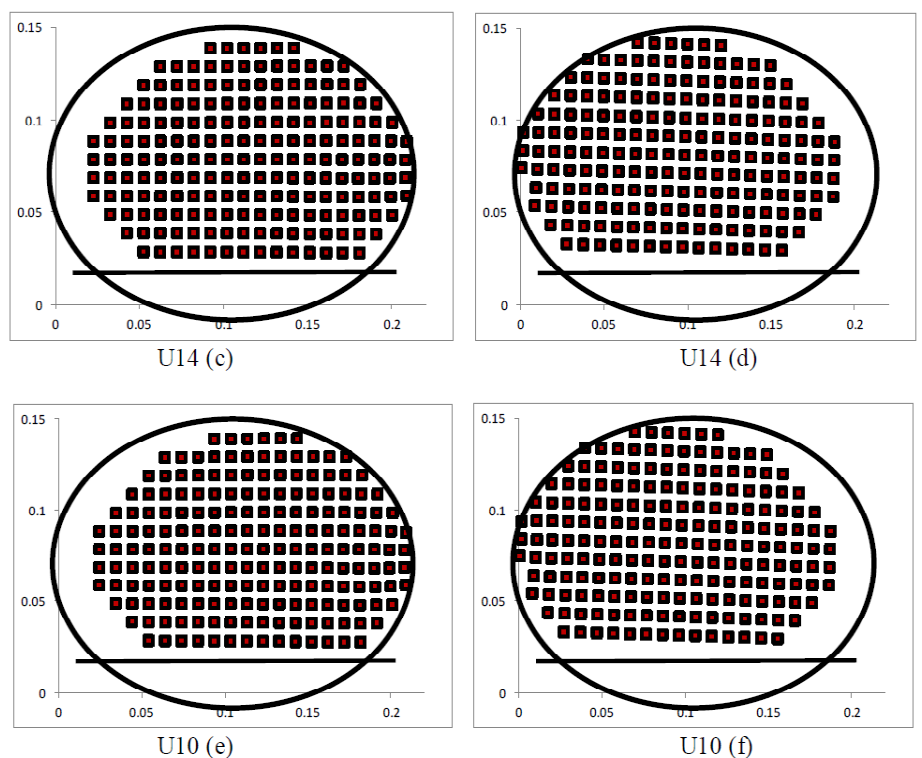

Figure 11: Snapshots of animations modeling the heading of lofted balls at near maximal speeds observed in actual games [38] using poor technique for adult Model 4 (a) and (b); U14 Model 10 (c) and (d); and U10 Model 16 (e) and (f). Frames on the left show initial maximal compression or coup. Frames on the right show subsequent maximal compression after rebound or contrecoup. Axis scales in meters. 

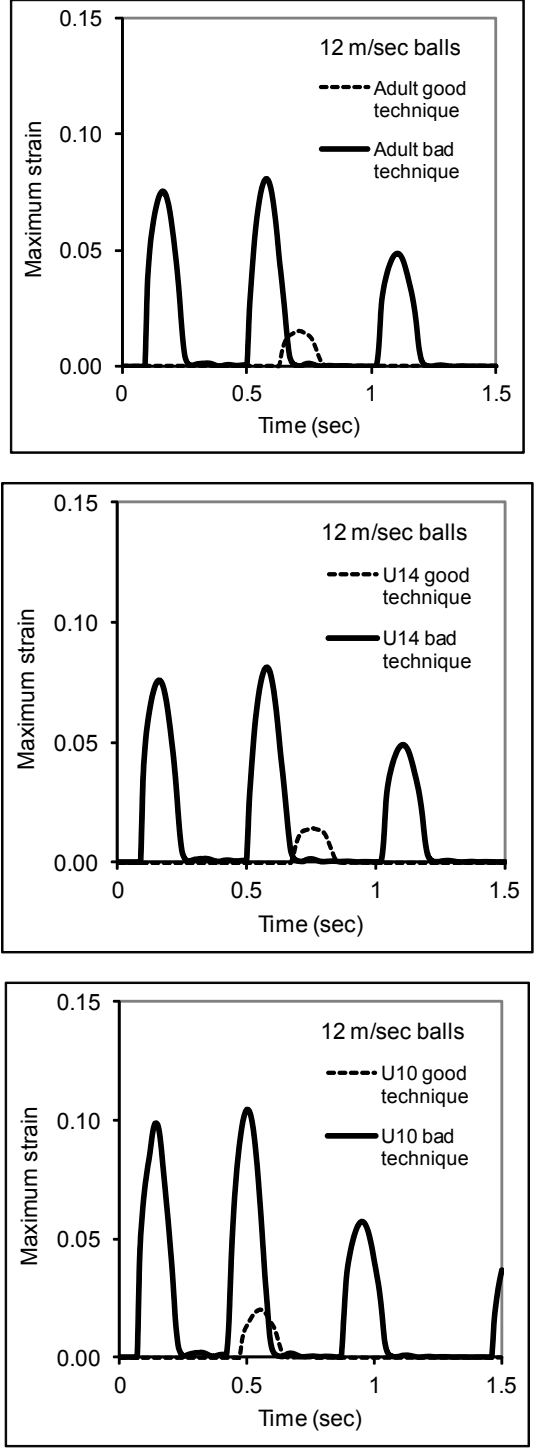

Figure 12: Time domain records of maximal strain during heading of high speed balls in adult models (top); U14 models (middle); U10 models (bottom).

When descending lofted balls at near maximum observed speed $(12 \mathrm{~m} / \mathrm{sec})$ are headed by an adult player running forward at $1 \mathrm{~m} / \mathrm{sec}$, a pattern similar to that seen with $7 \mathrm{~m} / \mathrm{sec}$ balls is observed. For example, the maximal compressive strain for adult Model 3, in this case is only 1.53 percent with $12 \mathrm{~m} / \mathrm{sec}$ balls, compared with 1.29 percent for adult Model 1 with $7 \mathrm{~m} / \mathrm{sec}$ balls. With poor technique, however, substantial deformation of the brain occurs over time in adult, teen, and youth players (Figures 11 and 12).

The most dangerous headed balls are rising balls that come directly off the foot, including shots headed away by a defender or and clearances that accidentally strike a player in front of goal.
These balls do not have time to be slowed by aerodynamic drag, and can reach $75 \mathrm{~m} / \mathrm{sec}$ in adult games [38]. If an adult player has time to prepare (less likely in these circumstance) and uses good technique, there are coup and countreoup impacts, with maximum compressive strains of 6.7 percent both at 0.18 and $0.63 \mathrm{sec}$ after impact (Figure 13, top). There is moderate brain deformation. Similar patterns are seen for teen and youth players (Figure 13, middle and bottom).

However, if such fast, rising balls are headed with poor technique, as can happen if the struck player does not see them coming or has insufficient time to tense neck and torso muscles, then clearly dangerous brain deformation can occur (Figure 14). There are multiple brain-skull collisions and substantial ringing (Figure 15). Such blows are clearly concussive in practice.
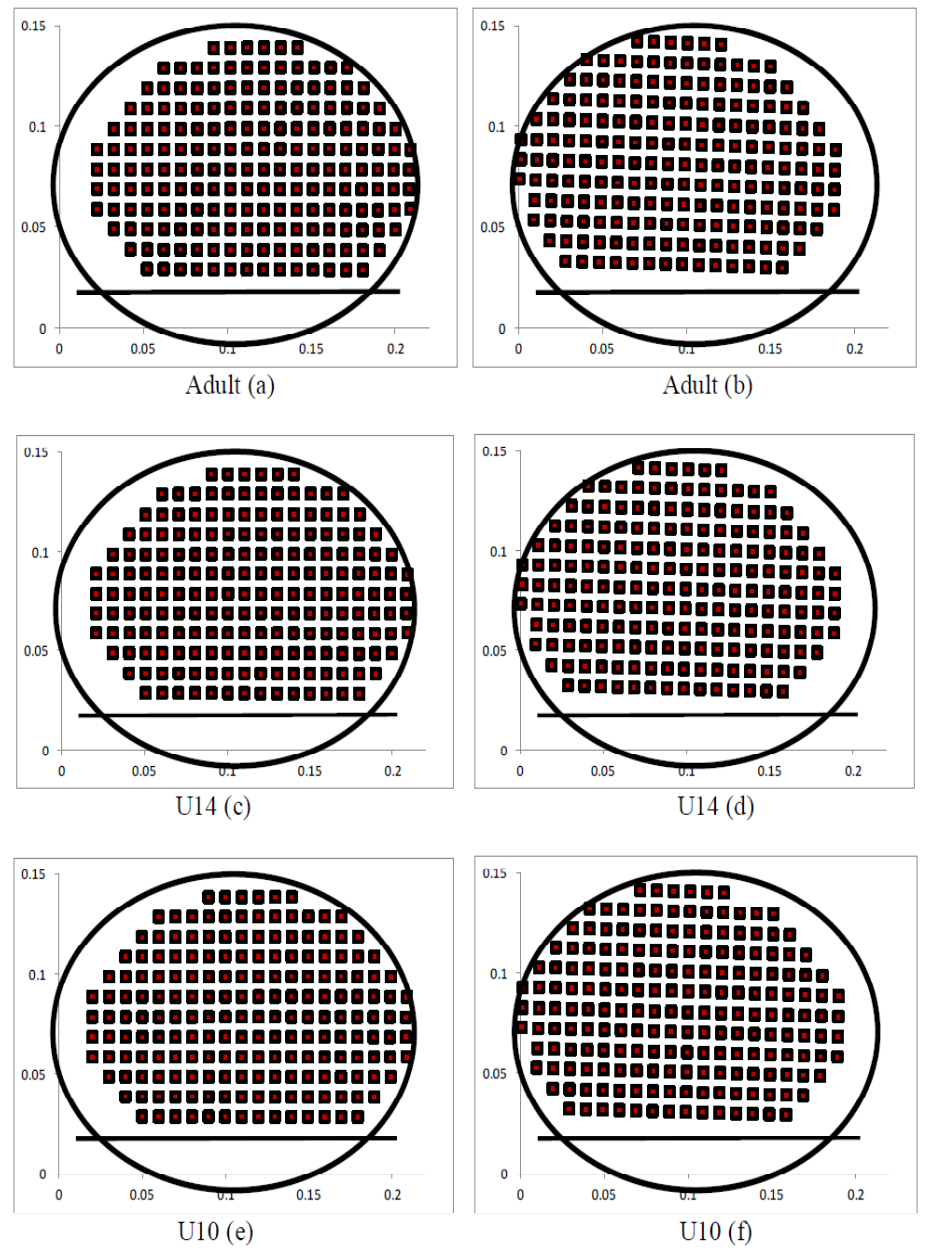

Figure 13: Snapshots of animations modeling the heading of rising balls at close range using good technique for adult Model 5 (a) and (b); U14 Model 11 (c) and (d); U10 Model 17 (e) and (f). Frames on the left show initial maximal compression or coup. Frames on the right show subsequent maximal compression after rebound or contrecoup. Axis scales in meters. 


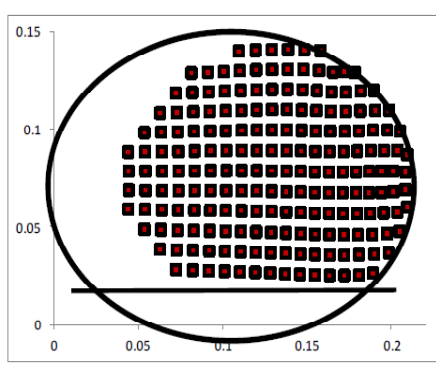

(a)

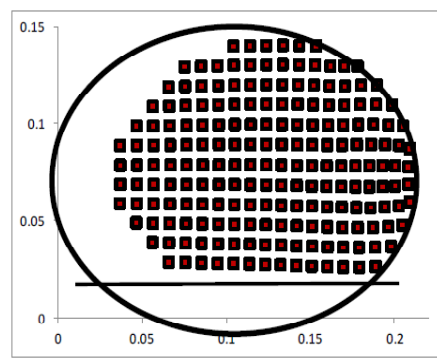

(c)

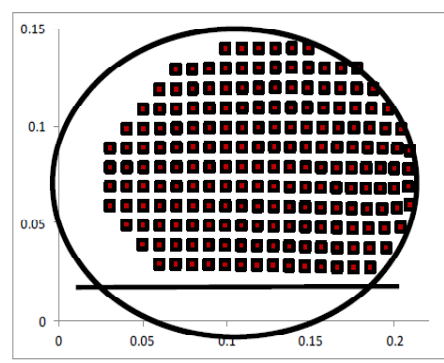

(e)

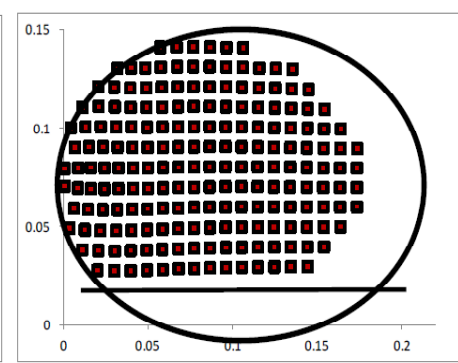

(b)

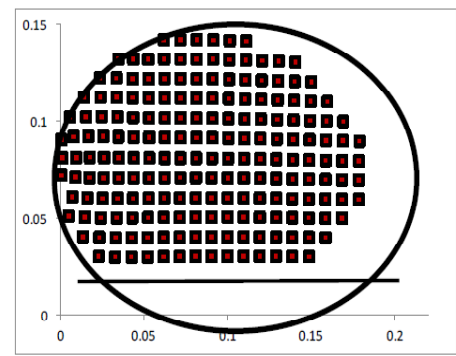

(d)

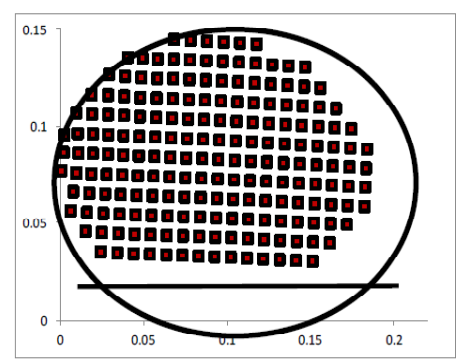

(f)
Figure 14: Snapshots of animations modeling the heading of rising balls at close range using poor technique for adult Model 6 (a) and (b); U14 Model 12 (c) and (d); U10 Model 18 (e) and (f). Frames on the left show initial maximal compression or coup. Frames on the right show subsequent maximal compression after rebound or contrecoup. Axis scales in meters.
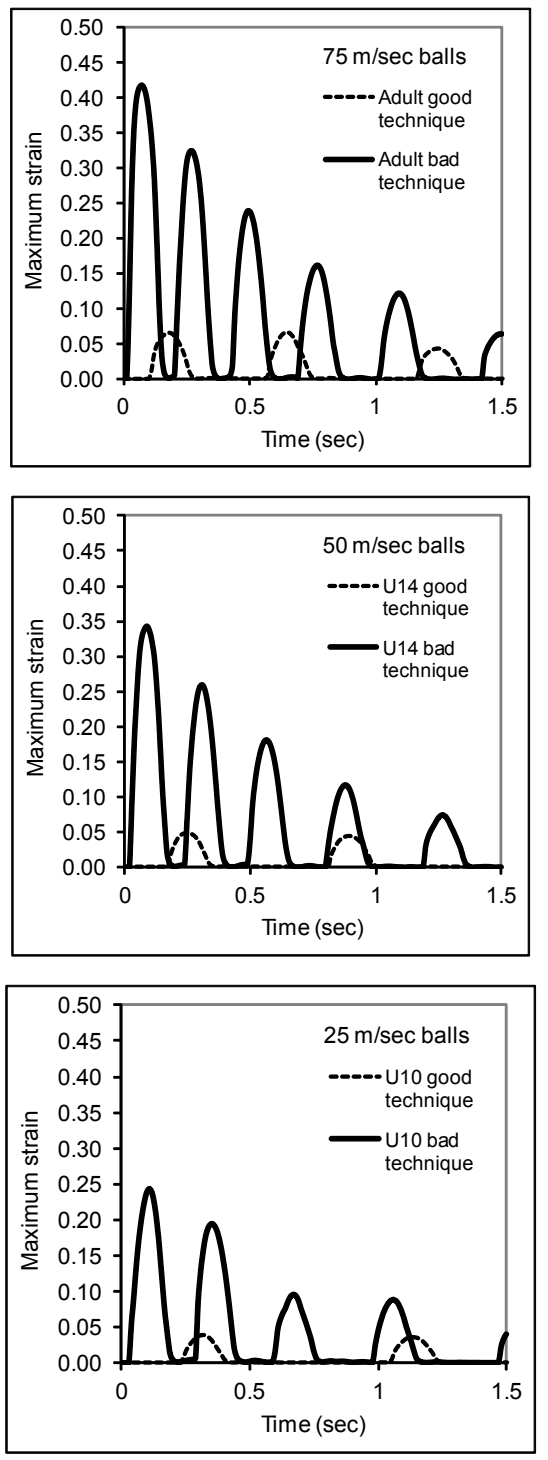

Figure 15: Time domain records of maximal strain during heading of high speed rising balls at close range in adult models (top); U14 models (middle); U10 models (bottom). Note extended scales for maximal strains. 


\section{Sensitivity Analysis for Stiffness and Damping}

Table 4 illustrates effects of stiffness and damping parameters on the maximal strains computed by the finite element method for Model 4, representing high speed lofted balls headed with poor technique in adults - a mid-spectrum model. The first row of the table shows the standard finite element model. Subsequent rows show halving or doubling of parameters. Halving of the Young's modulus of brain tissue toward the tail of the distribution of measured values [27] produces a roughly 30\% increase in maximum strain. Other modifications of parameters have more modest impact on brain deformation. The phenomena described in the present study are relatively insensitive to small changes in the assumed viscoelastic properties of the brain.

\begin{tabular}{|c|c|c|}
\hline E (Pa) & D (Pa-sec) & Max strain \\
\hline 10000 & 100 & 0.076 \\
\hline 5000 & & \\
\hline 20000 & 100 & 0.107 \\
\hline & 100 & 0.062 \\
\hline 10000 & & \\
\hline 10000 & 50 & 0.079 \\
\hline
\end{tabular}

Table 4: Sensitivity analysis for stiffness and damping (Model 4).

\section{Discussion}

Heading a soccer ball produces definite brain motion and deformation. The soft, viscoelastic brain, accelerates toward the rigid wall of the skull, deforms, and then rebounds, often traversing the fluid filled subarachnoid space and colliding with the opposite side of the skull in the classic coup and contrecoup pattern of closed head injury. During impacts with the inside of the skull the leading edges of the brain undergo local compression strain. The amount of local strain developed near the point of brain-skull contact is increased by force concentration that is caused by the mismatch between the curvature of the brain and the curvature of the overlying skull. The magnitude of the local compression strain varies with the incoming speed of the ball and with the technique of the player, quantified in terms of the effective body mass that the player can muster behind the ball by tensing muscles of the neck, chest, and abdomen. With good technique the head, neck, and torso are accelerated as a single unit. With poor technique the head is allowed to wobble to a significant degree, uncoupling the head from the torso and creating a much smaller effective mass. This smaller effective mass is much more easily accelerated by the reactive force produced during the head-ball collision. (Note that this type of low frequency motion and deformation is to be distinguished from that produced by shock or blast waves that propagate through solid bodies at sonic frequencies.)

The cerebrospinal fluid, in which the brain floats, acts imperfectly to protect the brain from acceleration injury. Because mass density of the brain is slightly greater than the mass density of the cerebrospinal fluid, the brain is not weightless in CSF, but instead experiences a degree of "artificial gravity" in response to whole head acceleration. As a result, the brain moves toward the inner wall of the rigid skull at the point of impact. The brain acts as a soft, viscoelastic body in this scenario and undergoes deformation upon striking the skull, characterized by compression, followed by rebound, and in many cases a secondary collision (contrecoup) with the opposite side of the skull. Greater whole head accelerations produce faster brain motion through the cerebrospinal fluid within the frame of reference of the skull, and in turn greater compressive strain, occurring sooner after head-ball contact, followed by quicker and more forceful rebound, and more intense contrecoup deformation.

For straight-on front-to-back impacts in heading, the parts of the brain that are compressed include the frontal lobes, which function in reasoning, personality, judgment, and self-control, and the occipital loves, which function in visual pattern recognition and interpretation. Surely, these structures and functions are highly important for humans, and need to be protected. The obvious question at this juncture is how much compression strain is dangerous or hazardous to the brain. Is it possible in any way to define a safe threshold for compressive strain? To determine safety experimentally one would need to study a very large number of subjects and to acquire very sensitive and specific measures of abnormal structure and function over a lifetime of exposure. Such experiments would be technically, and perhaps ethically, extremely difficult or impossible.

An amusing but insightful experiment of this kind in animals has been conducted by nature in the evolution by woodpeckers. These birds continually pound their beaks with vigor against trees to obtain food, to tunnel out nesting holes, and to signal their territories. High speed video studies of woodpecker head trajectories [45] show decelerations on the order of $10,000 \mathrm{~m} / \mathrm{sec}^{2}$ lasting about $0.5 \mathrm{msec}$ with each strike. Such impacts would induce a brain velocity in CSF of about $0.25 \mathrm{~m} / \mathrm{sec}$, which is equal to half the single blow concussive threshold in man, and somewhat less than the high speed, rising ball impacts of Models 6, 12, and 18 in Table $3(0.30$ to $0.38 \mathrm{~m} / \mathrm{sec})$ but greater than the changes in brain velocity caused by normal heading $(0.01$ to $0.07 \mathrm{~m} / \mathrm{sec})$. For an unconstrained one dimensional elastic body, an initial brain speed of $0.25 \mathrm{~m} / \mathrm{sec}$ with each blow would produce a compressive strain

of $\varepsilon^{*}=\mathrm{v}_{0} \sqrt{\rho / \mathrm{E}} \approx 0.25$, which would produce $12 \%$ lateral expansion, assuming a Poisson's ratio of 0.5 .

A tantalizing clue to the secret of the woodpecker's resistance to head acceleration is provided in Figure 2(a) of the published biomechanical study of Wang and coworkers [46], showing a high resolution micro-CT scan of a Great Spotted woodpecker's head in the coronal plane. This image is reproduced below as Figure 14 
and shows that the woodpecker's cerebral cortex is virtually flush against the interior of the skull. In most regions the subarachnoid space is reduced to a potential space less than 0.5 percent of the diameter of the brain. This narrow CSF gap in woodpeckers would severely limit brain deformation during front-to-back pounding. Assuming a Poisson's ratio of 0.5 and a coronal plane CSF width of less than 0.5 percent of brain diameter, the maximal compressive strain in front-to-back dimension for a woodpecker would be less than 1 percent, after which further brain deformation could not occur within the rigid skull. Thus, it would appear that woodpeckers have survived and thrived for a very long time suffering compressive strains of $1 \%$ or less. Using the woodpecker criterion, one might speculate that head-ball collisions that produce no more than $1 \%$ compressive strain in the major axis of the collision would be safe over the duration of a soccer playing career.

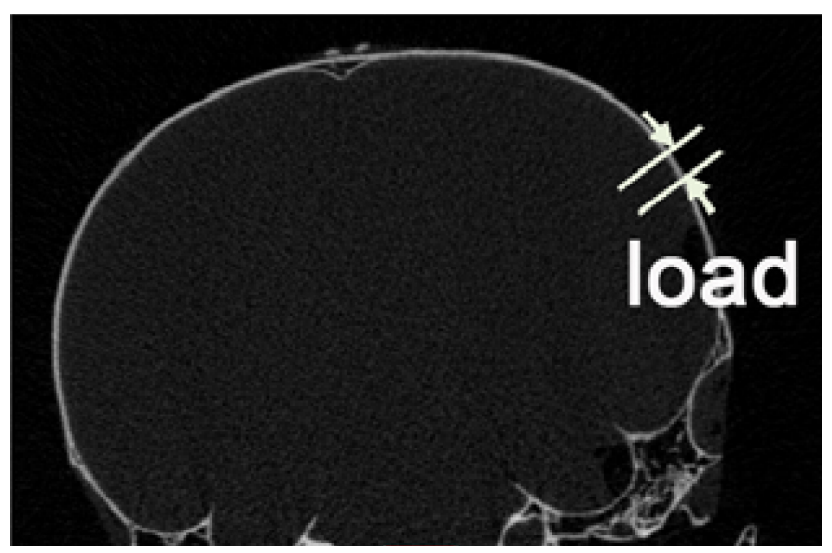

Figure 14: Reproduction of Figure 2(a) from Wang et al. [46], showing a high resolution micro-CT scan of a Great Spotted woodpecker's head in the coronal plane. In most regions the subarachnoid space is reduced to a potential space less than 0.5 percent of the diameter of the brain.

In the present study we find that a normal adult player using good technique, or a normal teen or youth player using good technique with age-appropriate balls, experiences just slightly greater than "safe" deformations of the brain according to the woodpecker criterion of less than $1 \%$ maximal compressive strain. However, deterioration toward poor technique leads to more hazardous brain compression and orthogonal expansion. In actual practice, most players will use less than perfect technique most of the time. In particular, under 10-year-old youth players using age appropriate balls but poor technique might experience frequent compressive strains greater than 6 percent. Impacts with rising balls, directly off the foot of the kicker, are especially problematic for players of any age.

Adjustments to the Laws of the Game to promote heading safety might include awarding a free kick to the opponent if a player strikes anyone in the head with a rising ball, reducing the legal mass of soccer balls of all sizes, especially the mass of the \#3 ball used by the youngest players, and never allowing water logged balls to be used in play. Requiring referees to weigh game balls routinely is not unreasonable.

Reduction of ball mass has a double benefit. Reduced mass reduces the momentum of the ball at any speed, which in turn reduces the momentum and change in velocity transferred to the head of the player. Reduced mass of the ball also allows aerodynamic drag (air resistance) to more easily slow lofted balls, which are the ones most commonly headed, to a slower incoming velocity, further reducing ball momentum at impact and the subsequent change in head velocity. Importantly for devotees of the game, lower mass balls would still allow near maximal speed when shooting the ball at goal in most cases, since most shots are taken at close range and are not long, lofted balls. Hence the ease of scoring would not be reduced significantly by lower mass balls. There would be some reduction in the longest possible passes achievable by adult, professional players, owing to a more pronounced effect of air resistance. At worst, this effect might encourage slightly more ball possession, build-up, and passing, which is not necessarily a bad outcome.

Should heading be banned or discouraged for younger players? This is a debatable proposition. If good technique is protective, then the sooner one learns good technique, the better. Here also lighter weight balls can help. The present analysis allows fairly straightforward simulation of likely results. Maximal compressive strain is proportional to initial brain velocity toward the skull, which is in turn proportional to change in head velocity during impact, which is in turn proportional to the ratio of ball mass to the effective mass of the player. Hence to make a $50 \%$ reduction in maximal compressive strain would require a roughly $50 \%$ reduction in ball mass. Maximum risk reduction would require eliminating heading from the game completely.

The present study is limited in several respects. The complex anatomy of the brain is reduced to a relatively simple geometric model in two dimensions. The viscoelastic properties of the brain are regarded as uniform, without distinction between gray and white matter. Straight on front-to-back impacts only are studied. Side impacts and twisting motion are not studied. Future research might well include more detailed anatomical models in three dimensions, including more exact external surface contours of the brain and internal surface contours of the skull.

Nevertheless, the present models appear to capture the essence of brain motion and deformation during soccer heading. They show that compressive strains on the order of $5 \%$ are to be expected occasionally in practical play and that obviously concussive strains of over $30 \%$ can occur in accidental situations. The importance of strong, proper heading technique that mounts a relatively large effective mass behind the ball is emphasized, and the advantages and tradeoffs of reduced ball mass for players of all are ages are discussed. 


\begin{tabular}{|l|l|l|}
\hline Symbol & Definition & Units \\
\hline$F_{x}$ & $\begin{array}{l}\text { Horizontal force applied to head of } \\
\text { player by ball }\end{array}$ & Newtons \\
\hline $\mathrm{L}$ & Body length & meters \\
\hline $\mathrm{m}$ & Mass of player & $\mathrm{kg}$ \\
\hline $\mathrm{m}^{\prime}$ & Effective mass of player & $\mathrm{kg}$ \\
\hline$\rho(\mathrm{h})$ & $\begin{array}{l}\text { Axial density of human body per unit } \\
\text { length as a function of height, h }\end{array}$ & $\mathrm{kg} / \mathrm{m}$ \\
\hline$\theta$ & $\begin{array}{l}\text { Angle of forward lean } \\
\text { Angular acceleration of the player's } \\
\text { body }\end{array}$ & $\mathrm{Rad} / \mathrm{sec}^{2}$ \\
\hline$\ddot{x}$ & $\begin{array}{l}\text { Acceleration of player's head in the } \\
\text { x-dimension as a result of impact with } \\
\text { the ball }\end{array}$ & $\mathrm{m} / \mathrm{sec}^{2}$ \\
\hline
\end{tabular}

Appendix 1: Concept of effective mass of a player.

The effective mass, $\mathrm{m}^{\prime}$, of the player is that mass which would require the same horizontal force to accelerate in the $\mathrm{x}$-dimension as does the total player in two or three dimensions. Here we assume temporarily excellent heading technique, in which the neck is strong and rigid and the head does not wobble. Consider first the grounded player sketched in text Figure 3, whose plant foot is fixed, whose body is free to pivot, and who leans into the shot with angle $\theta$. During contact $\theta$ will change by a relatively small value, in keeping with soccer playing experience. The horizontal force component, $F_{x}$, is applied by the ball to the player's head. By definition

$\mathrm{m}^{\prime}=\frac{\mathrm{F}_{\mathrm{x}}}{\ddot{\mathrm{x}}}=\frac{\mathrm{F}_{\mathrm{x}}}{\mathrm{L} \ddot{\theta} \sin \theta}$.

$F_{x}$ may be resolved into orthogonal components $F_{x} \cos (\theta)$ and $F_{x} \sin (\theta)$. For the grounded player $F_{x} \cos (\theta)$, which is parallel to the spine, does not induce body motion, because the spine is incompressible and the cleated boot prevents motion along this axis. Angular acceleration by $\mathrm{F}_{\mathrm{x}} \sin (\theta)$ is unconstrained, however.

Our strategy is to find a second expression for $\ddot{\theta}$ from Newton's second law and substitute into A1.1 to determine $\mathrm{m}^{\prime}$. Integrating force $=$ mass $\mathrm{x}$ acceleration for all small segments of mass along the length of the player's body and assuming that the angle of forward lean, $\theta$, changes only a small amount during impact, we have

$\mathrm{F}_{\mathrm{x}} \sin \theta=\ddot{\theta} \int_{0}^{\mathrm{L}} \mathrm{h} \rho(\mathrm{h}) \mathrm{dh}$.
Suppose, for example that $\rho(\mathrm{h})$ is a constant value, $\rho$; i.e. we model the player for simplicity as a slab (a more reasonable assumption than it may first appear). Then,

$$
\mathrm{F}_{\mathrm{x}} \sin \theta=\ddot{\theta} \rho \int_{0}^{\mathrm{L}} \mathrm{hdh}=\frac{1}{2} \ddot{\theta} \rho \mathrm{L}^{2} \text {, so that }
$$

$$
\ddot{\theta}=\frac{2 F_{x} \sin \theta}{\rho L^{2}}
$$

Substituting A1.4 into A1.1 and noting $\rho \mathrm{L}=\mathrm{m}$, the effective mass for the slab model is

$$
\mathrm{m}^{\prime}=\frac{\mathrm{m}}{2 \sin ^{2} \theta}
$$

Here, when $\theta=0$, the player is horizontal, and theoretically $\mathrm{m}^{\prime}=\infty$, because of the ground anchor. When $\theta=90$ degrees $\mathrm{m}^{\prime}=$ $\mathrm{m} / 2$, which fits with the expected leverage if all body mass were located at the center of mass of the slab.

We can also compute the effective mass of a player executing a jumping header for the slab model of the body. Now the $F_{x} \cos (\theta)$ force component induces downward translation of the slab along the $\theta$ axis, because there is no retarding contact with ground. The $F_{x} \sin (\theta)$ force component induces rotation about the center of mass of the slab. The effective mass retarding acceleration of the slab in the $\mathrm{x}$-dimension is given by

$\mathrm{m}^{\prime}=\frac{\mathrm{F}_{\mathrm{x}}}{\ddot{\mathrm{x}}_{\text {translational }}+\ddot{\mathrm{x}}_{\text {rotational }}}$.

By deduction from text Figure 3 in the absence of a ground anchor, the $\mathrm{x}$-component of translational acceleration is

$\ddot{\mathrm{x}}_{\text {translational }}=\frac{\mathrm{F}_{\mathrm{x}} \cos ^{2} \theta}{\mathrm{m}}$.

The $\mathrm{x}$-component of rotational acceleration is

$\ddot{x}_{\text {rotational }}=\frac{L}{2} \ddot{\theta} \sin \theta$,

and $\ddot{\theta}$ can be found from 
$\mathrm{F}_{\mathrm{x}} \sin \theta=2 \ddot{\theta} \rho \int_{0}^{\mathrm{L} / 2} \mathrm{hdh}=\ddot{\theta} \rho \frac{\mathrm{L}^{2}}{4}$.

Solving A1.9 for $\ddot{\theta}$ and combining A1.6 through A1.8 yields

$$
\mathrm{m}^{\prime}=\frac{\mathrm{m}}{2 \sin ^{2} \theta+\cos ^{2} \theta}
$$

Thus when $\theta \approx 0$, the player is horizontal, and $\mathrm{m}^{\prime} \approx \mathrm{m}$, as expected for a perfect diving header with the player suspended in air. When $\theta \approx 90$ degrees $\mathrm{m}^{\prime} \approx \mathrm{m} / 2$, the same as for the anchored slab model, as expected. For a reasonable playing value of $\theta=\tan ^{-1}(4)$, representing the good technique of a player striking the ball with slight forward lean, the effective mass is 0.53 for the grounded player model and 0.515 for the jumping player model. Hence for routine computation of excellent technique we can estimate $m^{\prime}=m / 2$ to represent a player whose neck is strong and stiff (perfect technique) at impact. If the neck is allowed to wobble at impact (extremely poor technique), the effective mass approaches the mass of the head only. Thus a range of effective masses between these limits can represent a range of techniques. In this way we have a simple mathematical model of the performance of a wide range of complex mechanical linkages of the actual human body in action.

How different from a slab is a person in terms of moment of inertia and effective mass? Using anatomically realistic estimates of cross sectional areas of bone and water density in an adult human body (data not shown) and the definite integral A1.2, the author found an anatomically realistic effective mass that differed less than 5 percent from that of the slab model. In terms of moments of inertia, people are rather like slabs. They have above average mass in the abdomen near the body's center of mass, and below average mass elsewhere, which yields an overall effective mass much like that of a slab.

\begin{tabular}{|l|l|}
\hline A & cross sectional area of brain model \\
\hline a & time averaged, brief, forceful acceleration \\
\hline c & strain wave propagation speed in an elastic rod \\
\hline D & damping or loss modulus of brain tissue \\
\hline E & Young's modulus of elasticity of brain tissue \\
\hline$\varepsilon$ & local strain in a model of the brain \\
\hline$\varepsilon^{*}$ & a threshold harmful compressive strain \\
\hline
\end{tabular}

\begin{tabular}{|l|l|}
\hline $\mathrm{k}$ & $\begin{array}{l}\text { spring constant of a dx length column of elastic material, } \\
\text { namely } \mathrm{k}=\overline{\mathrm{A}} \mathrm{E} / \mathrm{dx}\end{array}$ \\
\hline $\mathrm{L}$ & $\begin{array}{l}\text { length of column of elastic material in brain model along the } \\
\text { axis of linear acceleration }\end{array}$ \\
\hline$\mu$ & $\begin{array}{l}\text { damping constant of a dx length column of elastic material, } \\
\text { namely } \mu=\mathrm{AD} / \mathrm{dx}\end{array}$ \\
\hline$v$ & Poisson's ratio \\
\hline$\rho_{\mathrm{CSF}}$ & mass density of cerebrospinal fluid \\
\hline$\rho$ & mass density of brain \\
\hline $\mathrm{t}$ & time \\
\hline$\theta$ & angle \\
\hline$\dot{\theta}$ & angular velocity \\
\hline$\ddot{\theta}$ & angular acceleration \\
\hline $\mathrm{v}$ & velocity \\
\hline $\mathrm{V}$ & velocity of brain toward skull at instant of brain-skull impact \\
\hline $\mathrm{V}$ & volume \\
\hline $\mathrm{y}$ & tongitudinal distance along axis of initial acceleration \\
\hline
\end{tabular}

\section{Appendix 2: Nomenclature.}

\section{References}

1. Bennett JC, Plum F (1996) Cecil textbook of medicine 1.

2. JordanBD (1987)Neurologicaspects ofboxing.Arch Neurol44:453-459.

3. Bohnen N, Twijnstra A, Jolles J (1992) Performance in the stroop color word test in relationship to the persistence of symptoms following mild head injury. Acta Neurol Scand 85: 116-121.

4. Cronwall D, Wrightson P (1975) Cumulative effect of concussion. Lancet 2: 995-997.

5. Sortland O, Tysvaer AT (1989) Brain damage in former association football players. An evaluation by cerebral computed tomography. Neuroradiology 31: 44-48.

6. Tysvaer AT, Storli OV, Bachen NI (1989) Soccer injuries to the brain. A neurologic and electroencephalographic study of former players. Acta Neurol Scand 80: 151-156.

7. MatserJT,KesselsAG, JordanBD, LezakMD, TroostJ(1998)Chronictraumatic brain injury in professional soccer players. Neurology 51: 791-796. 
8. Tysvaer AT, Lochen EA (1991) Soccer injuries to the brain. A neuropsychologic study of former soccer players. Am J Sports Med 19: 56-60.

9. Barnes BC, Cooper L, Kirkendall DT, McDermott TP, Jordan BD, et al. (1998) Concussion history in elite male and female soccer players. Am J Sports Med 26: 433-438.

10. Dailey SW, Barsan WG (1992) Head injuries in soccer: A case for protective headgear? The Physician and Sportsmedicine 20: 79-85.

11. Boden BP, Kirkendall DT, Garrett WE (1998) Concussion incidence in elite college soccer players. Am J Sports Med 26: 238-241.

12. Babbs CF (2000) Brain injury in amateur soccer players. Journal of the American Medical Association 283: 882-883.

13. Matser EJ, Kessels AG, Lezak MD, Jordan BD, Troost J(1999) Neuropsychological impairment in amateur soccer players. JAMA 282: 971-973.

14. Master J, Kessels A, Lezak M, Troost J (2010) A dose-response relation of headers and concussions with cognitive impairment in professional soccer players. Journal of Clinical and Experimental Neuropsychology 23: 770-774.

15. Lipton M, Kim N, Zimmerman M, Stewart W, Branch C, et al. (2013) Soccer heading is associated with white matter microstructural and cognitive abnormalities. Radiology 268: 850-857.

16. Koerte I, Ertl-Wagner B, Reiser M, Zafonte R, Shenton M (2012) White matter integrity in the brains of professional soccer players without a symptomatic concussion. JAMA 308: 1859-1861.

17. DiVirgilio T, Hunter A, Wilson L, Stewart W, Goodall S, et al. (2016) Evidence for acute electrophysiological and cognitive changes following routine soccer heading. EBioMedicine 13: 66-71.

18. Witol A, Webbe F (1994) Neuropsychological deficits associated with soccer play. Archives of Clinical Neuropsychology 9: 204-205.

19. Jordan SE, Green GA, Galanty HL, Mandelbaum BR, J (1996) bour BA. Acute and chronic brain injury in united states national team soccer players. The American Journal of Sports Medicine 24: 205-210.

20. Kontos A, Dolese A, Elbin R, Covassin T, Warren B (2011) Relationship of soccer heading to computerized neurocognitive performance and symptoms among female and male youth soccer players. Brain Injury 12: 1234-1241.

21. Asken JJ, Schwartz RC (1998) Heading the ball in soccer: What's the risk of brain injury? The Physician and Sportsmedicine 26: 37-44.

22. Versace $J$ (1971) A review of the severity index. Proceedings of the 15th Stapp car crash conference 771-796.

23. Pudenz RH, Shelden CH (1946) The lucite calvarium-a method for direct observation of the brain. Journal of Neurosurgery 3: 487-505.

24. Gosch HH, Gooding E, Schneider RC (1969) Distortion and displacement of the brain in experimental head injuries. Surg Forum 20: 425426.

25. Gosch HH, Gooding E, Schneider RC (1970) The lexan calvarium for the study of cerebral responses to acute trauma. J Trauma 10: 370376.

26. Ommaya AK (1966) Experimental head injury in the monkey. In: Caveness WF, Walker AE, eds. Head injury. Philadelphia: J B lippincott Company 260-275.
27. Babbs CF (2005) Brain motion and deformation during closed head injury in the presence of cerebrospinal fluid. Journal of Mechanics in Medicine and Biology 5: 277-306.

28. Margulies SS, Thibault LE (1989) An analytical model of traumatic diffuse brain injury. Biomechanical of Engineering 111: 241-249.

29. Meaney DF, Smith DH, Shreiber DI, Bain AC, Miller RT, et al. (1995) Biomechanical analysis of experimental diffuse axonal injury. J Neurotrauma 12: 689-694.

30. Ljung $C$ (1975) A model for brain deformation due to rotation of the skull. Biomechanics 8: 263-264.

31. Bycroft GN (1973) Mathematical model of a head subjected to an angular acceleration. J Biomech 6: 487-495.

32. Paris A (1882) Subtle is the lord-the science and the life of albert einstein. Oxford and New York: Oxford University Press.

33. Shigeno T, Brock M, Shigeno S, Fritschka E, Cervos-Navarro J (1982) The determination of brain water content: Microgravimetry versus drying-weighing method. J Neurosurg 57: 99-107.

34. DiResta G, Lee J, Arbit E (1991) Measurement of brain tissue specific gravity using pycnometry. Neuroscience 39: 245-251.

35. Duck FA (1990) Physical properties of tissue-a comprehensive reference book. London: Academic Press.

36. Official rules of soccer (1994) Chicago: Triumph Books.

37. The laws of the game (2000) 2000.

38. Babbs CF (2001) Biomechanics of heading a soccer ball: Implications for player safety. Scientific World Journal 1: 281-322.

39. Brekhovskikh LM, Goncharov V (1994) Mechanics of continua and wave dynamics. Berlin: Springer-Verlag.

40. Hagedorn P, DasGupta A (2007) Vibrations and waves in continuous mechanical systems. Wiley-Science.

41. Miller K, Chinzei K (1997) Constitutive modelling of brain tissue: Experiment and theory. J Biomech 30: 1115-1121.

42. Nellhaus $G$ (1968) Head circumference from birth to eighteen years. Pediatrics 41: 106-114.

43. Fung YC (1981) Biomechanics: Mechanical properties of living tissues. New York: Springer-Verlag.

44. Varnes D (1962) Analysis of plastic deformation according to von mises' theory with application to the south silverton area, san juan county colorado. U. S. Geological Survey Professional Paper 387-B: B1-B49.

45. May PR, Fuster JM, Haber J, Hirschman A (1979) Woodpecker drilling behavior. An endorsement of the rotational theory of impact brain injury. Arch Neurol 36: 370-373.

46. Wang L, Cheung J, Pu F, Li D, Zhang M, et al. (2011) Why do woodpeckers resist head impact injury: A biomechanical investigation. PLoS One 6: e26490. 